\title{
Leitlinienreport der S3-Leitlinie Diagnostik und Therapie der Plattenepithelkarzinome und Adenokarzinome des Ösophagus
}

Version 2.0 - Dezember 2018, AWMF-Registernummer: 021/023OL

Autoren

Rainer Porschen ${ }^{1}$, Thomas Langer ${ }^{2}$, Pia van Leeuwen ${ }^{3}$

Institute

1 Klinik für Innere Medizin, Klinikum Bremen Ost

2 Leitlinienprogramm Onkologie der Deutschen Krebsgesellschaft, Berlin

3 Deutsche Gesellschaft für Verdauungs- und Stoffwechselkrankheiten, Berlin

Bibliografie

DOI https://doi.org/10.1055/a-0833-6106

Z Gastroenterol 2019; 57: e103 - e119

(c) Georg Thieme Verlag KG, Stuttgart · New York

ISSN 0044-2771
Korrespondenzadresse

Prof. Dr. med. Rainer Porschen

Chefarzt der Klinik für Innere Medizin, Klinikum Bremen Ost, Züricher Str. 40, D-28325 Bremen

Tel.: ++49/421/4081221

Fax: $++49 / 421 / 4082234$

rainer.porschen@klinikum-bremen-ost.de

\begin{tabular}{|c|c|c|}
\hline \multicolumn{2}{|c|}{ Inhaltsverzeichnis } & \multirow{2}{*}{$\begin{array}{l}\text { Seite } \\
\text { e103 }\end{array}$} \\
\hline 1. & Informationen zum Leitlinienreport & \\
\hline 1.1. & Autoren des Leitlinienreports & e104 \\
\hline 1.2. & Herausgeber & e104 \\
\hline 1.3. & Federführende Fachgesellschaft(en) der Leitlinie & e104 \\
\hline 1.4. & Finanzierung der Leitlinie & e104 \\
\hline 1.5. & Kontakt & e104 \\
\hline 1.6. & Zitierweise des Leitlinienreports & e104 \\
\hline 1.7. & Weitere Dokumente zur Leitlinie & e104 \\
\hline 1.8. & Verwendete Abkürzungen & e104 \\
\hline 2. & Geltungsbereich und Zweck der Leitlinie & e105 \\
\hline 2.1. & Zielsetzung, Fragestellung und Adressaten & e105 \\
\hline 2.2. & Gültigkeitsdauer und Aktualisierungsverfahren & e105 \\
\hline 3. & Zusammensetzung der Leitliniengruppe & e105 \\
\hline 4. & $\begin{array}{l}\text { Aktualisierung der Fragestellungen und } \\
\text { Einschlusskriterien }\end{array}$ & e105 \\
\hline 5. & Methodisches Vorgehen & e105 \\
\hline 5.1. & Leitlinienadaptation & e105 \\
\hline 5.2. & Systematische Literaturrecherchen & e106 \\
\hline 5.2.1. & Suche und Auswahl der Evidenz & e106 \\
\hline 5.2.2. & Bewertung und Extraktion der Evidenz & e106 \\
\hline 5.3. & $\begin{array}{l}\text { Formulierung der Empfehlungen und formale } \\
\text { Konsensusfindung }\end{array}$ & e107 \\
\hline
\end{tabular}

\begin{tabular}{|l|l|l|}
\multicolumn{2}{|l|}{ Inhaltsverzeichnis } & Seite \\
\hline 5.3.1. & Schema der Empfehlungsgraduierung & $\mathrm{e} 107$ \\
\hline 5.3.2. & Festlegung des Empfehlungsgrades & $\mathrm{e} 107$ \\
\hline 5.3.3. & $\begin{array}{l}\text { Formale Konsensusverfahren und Konsensus- } \\
\text { konferenz }\end{array}$ & $\mathrm{e} 107$ \\
\hline 6. & Ableitung der Qualitätsindikatoren & $\mathrm{e} 107$ \\
\hline 7. & Reviewverfahren und Verabschiedung & $\mathrm{e} 108$ \\
\hline 8. & $\begin{array}{l}\text { Unabhängigkeit und Umgang mit Interessen- } \\
\text { konflikten }\end{array}$ & $\mathrm{e} 108$ \\
\hline 9. & Verbreitung und Implementierung & $\mathrm{e} 109$ \\
\hline 10. & Tabellenverzeichnis & $\mathrm{e} 109$ \\
\hline 11. & Anlagen & $\mathrm{e} 109$ \\
\hline 11.1. & Formblatt der AWMF zur Erklärung von Interes- & $\mathrm{e} 109$ \\
\hline 11.2. & Ergebnisse der Interessenkonflikterklärungen & $\mathrm{e} 112$ \\
\hline Literatur & & $\mathrm{e} 119$ \\
\hline & & \\
\hline
\end{tabular}

\section{Informationen zum Leitlinienreport}

Dieser Leitlinienreport dokumentiert das Aktualisierungsverfahren der Leitlinie von 2017 - 2018 (Version 2). Der Leitlinienreport zur Erstellung der Version 1 kann unter https://www.leitlinienprogramm-onkologie.de/leitlinien/oesophaguskarzinom/) eingesehen werden. 


\subsection{Autoren des Leitlinienreports}

Prof. Dr. med. Rainer Porschen, Dipl.-Soz.-Wiss.Thomas Langer, Pia van Leeuwen

\subsection{Herausgeber}

Leitlinienprogramm Onkologie der Arbeitsgemeinschaft der Wissenschaftlichen Medizinischen Fachgesellschaften e. V. (AWMF), der Deutschen Krebsgesellschaft e. V. (DKG) und der Deutschen Krebshilfe (DKH).

\subsection{Federführende Fachgesellschaft(en) der Leitlinie}

Deutsche Gesellschaft für Gastroenterologie, Verdauungs- und Stoffwechselkrankheiten (DGVS)

\section{${ }^{1913}$ DGVS \\ Deutsche Gesellschaft für \\ Gastroenterologie, \\ Verdauungs- und \\ Stoffwechselkrankheiten}

\subsection{Finanzierung der Leitlinie}

Die Erstellung und Aktualisierung der Leitlinie in 2017 - 2018 wurde von der Deutschen Krebshilfe im Rahmen des Leitlinienprogramms Onkologie gefördert.

\subsection{Kontakt}

Office Leitlinienprogramm Onkologie

c/o Deutsche Krebsgesellschaft e. V.

Kuno-Fischer-Straße 8

14057 Berlin

leitlinienprogramm@krebsgesellschaft.de

www.leitlinienprogramm-onkologie.de

\subsection{Zitierweise des Leitlinienreports}

Leitlinienprogramm Onkologie (Deutsche Krebsgesellschaft, Deutsche Krebshilfe, AWMF): S3 Leitlinie zur Diagnostik und Therapie der Plattenepithelkarzinome und Adenokarzinome des Ösophagus, Leitlinienreport 2.0, 2018, AWMF Registernummer: 021/ 0230L, https://www.leitlinienprogramm-onkologie.de/leitlinien/ oesophaguskarzinom/)

\subsection{Weitere Dokumente zur Leitlinie}

Die Leitlinie liegt als Lang- und Kurzversion vor. Außerdem gibt es eine Patientenleitlinie (Laienversion der Leitlinie) sowie zusätzliche Dokumente zur Evidenzrecherche (Evidenzberichte). Alle Dokumente zur Leitlinie sind über die folgenden Seiten zugänglich:

- AWMF (https://www.awmf.org/leitlinien/detail/II/021-023OL. html)

- Leitlinienprogramm Onkologie https://www.leitlinienprogramm-onkologie.de/leitlinien/oesophaguskarzinom/

- Deutsche Gesellschaft für Gastroenterologie, Verdauungs- und Stoffwechselkrankheiten (DGVS) (www.dgvs.de)

- Guidelines International Network (www.g-i-n.nets)

\subsection{Verwendete Abkürzungen}

\begin{tabular}{|c|c|}
\hline Abkürzung & Erläuterung \\
\hline AG & Arbeitsgruppe \\
\hline AWMF & $\begin{array}{l}\text { Arbeitsgemeinschaft der Wissenschaftlichen } \\
\text { Medizinischen Fachgesellschaften }\end{array}$ \\
\hline AWMF-IMWi & AWMF-Institut für Medizinisches Wissensmanagement \\
\hline BDP & Bundesverband deutscher Pathologen \\
\hline BNG & $\begin{array}{l}\text { Berufsverband der Niedergelassenen Gastroenterologen } \\
\text { Deutschlands }\end{array}$ \\
\hline CAO-V & $\begin{array}{l}\text { Chirurgische Arbeitsgemeinschaft Onkologie in der } \\
\text { Deutschen Gesellschaft für Allgemein- und } \\
\text { Viszeralchirurgie }\end{array}$ \\
\hline DEGRO & Deutsche Gesellschaft für Radioonkologie \\
\hline DELBI & Deutsches Leitlinienbewertungsinstrument \\
\hline DGAV & $\begin{array}{l}\text { Deutsche Gesellschaft für Allgemein- und } \\
\text { Viszeralchirurgie }\end{array}$ \\
\hline DGCH & Deutsche Gesellschaft für Chirurgie \\
\hline DGE & Deutsche Gesellschaft für Ernährung \\
\hline DGEM & Deutsche Gesellschaft für Ernährungsmedizin \\
\hline DGHO & Deutsche Gesellschaft für Hämatologie und Onkologie \\
\hline DGIM & Deutsche Gesellschaft für Innere Medizin \\
\hline DGKL & $\begin{array}{l}\text { Deutsche Vereinte Gesellschaft für Klinische Chemie } \\
\text { und Laboratoriumsmedizin }\end{array}$ \\
\hline DGN & Deutsche Gesellschaft für Nuklearmedizin \\
\hline DGP & Deutsche Gesellschaft für Pathologie \\
\hline DGVS & $\begin{array}{l}\text { Deutsche Gesellschaft für Verdauungs- und Stoff- } \\
\text { wechselkrankheiten }\end{array}$ \\
\hline DKG & Deutsche Krebsgesellschaft \\
\hline $\mathrm{DKH}$ & Deutsche Krebshilfe \\
\hline DKV & Deutsche Krankenversicherung \\
\hline DRG & Deutsche Röntgengesellschaft \\
\hline EK & Expertenkonsens \\
\hline IQWIG & $\begin{array}{l}\text { Institut für Qualität und Wirtschaftlichkeit im } \\
\text { Gesundheitswesen }\end{array}$ \\
\hline LA & Leitlinienadaptation \\
\hline LoE & Level of Evidence \\
\hline MDK & Medizinischer Dienst der Krankenversicherung \\
\hline $\mathrm{OL}$ & Leitlinienprogramm Onkologie \\
\hline $\mathrm{PET} / \mathrm{CT}$ & $\begin{array}{l}\text { Positronen-Emissions-Tomographie/ } \\
\text { Computertomographie }\end{array}$ \\
\hline $\mathrm{PICO}$ & Population, Intervention, Comparison, Outcome \\
\hline PSO & $\begin{array}{l}\text { Arbeitsgemeinschaft für Psychosoziale Onkologie in } \\
\text { der Deutschen Krebsgesellschaft }\end{array}$ \\
\hline $\mathrm{RCT}$ & Randomized Controlled Trial \\
\hline SR & Systematic Review \\
\hline TNM & für engl. Tumor, Node, Metastasis \\
\hline TSE & turbo spin-echo \\
\hline UICC & UICC(Union internationale contre le cancer)-Klassifikation \\
\hline ZVK & Deutscher Verband für Physiotherapie \\
\hline
\end{tabular}




\section{Geltungsbereich und Zweck der Leitlinie}

\subsection{Zielsetzung, Fragestellung und Adressaten}

Die Ziele der S3-Leitlinie, die Adressaten sowie die bearbeiteten Fragestellungen sind in der Langversion der Leitlinie beschrieben.

\subsection{Gültigkeitsdauer und Aktualisierungsverfahren}

Die S3-Leitlinie in der Version 2 ist bis zur nächsten Aktualisierung gültig, maximal aber 5 Jahre. Es ist vorgesehen, die Inhalte der Leitlinie im Rahmen eines sogenannten „Living-guideline-Ansatzes“ jährlich auf Basis aktueller Studiendaten und neuer Publikationen sowie Rückmeldungen aus der Leitliniengruppe zu prüfen und gegebenenfalls zu aktualisieren. Hierzu wird einmal jährlich eine systematische Literaturrecherche und Evidenzbeurteilung durchgeführt.

Kommentare und Hinweise für den Aktualisierungsprozess sind ausdrücklich erwünscht und können an: oesophaguskarzinom@ leitlinienprogramm-onkologie.de adressiert werden.

\section{Zusammensetzung der Leitliniengruppe}

Die Leitliniengruppe war multidisziplinär und multiprofessionell unter direkter Beteiligung von Patientenvertretern zusammengesetzt. Der Aktualisierungsprozess wurde maßgeblich von der Steuergruppe geplant und umgesetzt. Alle beteiligten Personen und Organisationen, die an der Aktualisierung der Leitlinie beteiligt waren, sind in der Langversion aufgeführt.

\section{Aktualisierung der Fragestellungen und Einschlusskriterien}

Die Grundstruktur der Leitlinie basiert auf der Einteilung in Hauptkomplexe, die mit den Kapiteln der vorliegenden Leitlinie korrespondieren. Auf der Grundlage der bestehenden Schlüsselfragen (Version 1) wurden die Schlüsselfragen zu den einzelnen Themenblöcken von der Steuergruppe bei einem Treffen am 5.7.2017 diskutiert und insbesondere die Ein- und Ausschlusskriterien bei einigen Schlüsselfragen überarbeitet. Anschließend wurde das Protokoll der Sitzung an die gesamte Leitliniengruppe versendet, mit der Bitte um Ergänzungen und ggf. Korrekturen.

Im Rahmen des Prozesses wurden die folgenden Änderungen und Ergänzungen beschlossen:

\section{Änderungen der Einschlusskriterien}

AG1 Risikofaktoren, Prävention, Screening (vorher nur systematische Reviews):

- Risikofaktoren: Observationsstudien mit $n \geq 500$

- Prävention: RCT mit $n \geq 50$

AG2 Diagnostik, Pathologie (vorher systematische Reviews oder Einschluss mindestens kontrollierter Primärstudien mit mindestens 50 Patienten, wenn keine aggregierte Evidenz vorhanden):

- Meta-Analysen, keine systematischen Reviews,

- $R C T n \geq 50$

- Prospektive Kohortenstudien mit $n \geq 500$
AG3 Kurativ intendierte Therapie (vorher systematische Reviews oder Einschluss mindestens kontrollierter Primärstudien mit mindestens 50 Patienten, wenn keine aggregierte Evidenz vorhanden):

- RCT mit $n \geq 50$

AG 4 Palliation (vorher systematische Reviews oder Einschluss mindestens kontrollierter Primärstudien mit mindestens 20 Patienten, wenn keine aggregierte Evidenz vorhanden):

- RCT mit $n \geq 50$

\section{Änderungen/Neuerungen bei den Schlüsselfragen}

Arbeitsgruppe 1: Risikogruppen Prävention Screening

- Überwachungs-ÖGD bei Risikogruppen: wird Expertenkonsens, Verweis auf die alte Leitlinie

Arbeitsgruppe 2: Primärdiagnostik, Diff.-Diagnostik inkl. Pathologie:

- Stellenwert der Abdomensonographie: De-novo-Recherche

- MSI-Status: De-novo-Recherche

Arbeitsgruppe 3: Kurativ intendierte Therapie

- Präoperative Vorbereitung: wird gestrichen

- Verbessert eine präoperative Chemo-/Radiochemotherapie das Überleben: PCR-Rate wird mit aufgenommen

- Rolle des PET-CT in der Remissionsvorhersage: Bezug auf IQWIG-Bericht

Arbeitsgruppe 4: Palliation

- Multidiziplinäre Therapie: wird als systematische Recherche nicht weitergeführt, weitere Bearbeitung im Expertenkonsens

- Andere endoskopische Verfahren: Stent als Intervention hinzugefügt

- Nachsorge und Rehabilitation: wird als systematische Recherche nicht weitergeführt, weitere Bearbeitung im Expertenkonsens

Zusätzlich wurde im Oktober 2017 eine Recherche zum Thema Ultraschall-Diagnostik bei Ösophaguskarzinom-Patienten mit Verdacht auf Lebermetastasen durchgeführt.

\section{Methodisches Vorgehen}

\subsection{Leitlinienadaptation}

Zur Identifizierung relevanter deutscher und englischer Leitlinien zur Diagnostik und Therapie des Ösophaguskarzinoms wurde eine Leitlinienrecherche durchgeführt. Die Suche ergab ingesamt 9 potentiell relevante Leitlinien, die aber überwiegend (8 von 9) den methodischen Anforderungen nicht genügten. Letztendlich entschied sich die Leitliniengruppe im Aktualisierungsverfahren, keine Empfehlungen aus der vorliegenden evidenzbasierten Leitlinie zu übernehmen, da diese nicht mehr als aktuell angesehen wurden. Die Leitlinienrecherche und -bewertung ist ausführlich im Methodenreport der CGS-Usergroup dargestellt (verfügbar unter: https://www.leitlinienprogramm-onkologie.de/leitlinien/ oesophaguskarzinom/). 
- Tab. 1 Schema der Evidenzgraduierung nach Oxford (Version März 2009).

\begin{tabular}{|c|c|c|c|c|}
\hline Level & $\begin{array}{l}\text { Therapy/ Prevention, } \\
\text { Aetiology/Harm }\end{array}$ & Prognosis & Diagnosis & $\begin{array}{l}\text { Differential diagnosis/ } \\
\text { symptom prevalence study }\end{array}$ \\
\hline $1 a$ & SR (with homogeneity) of RCTs & $\begin{array}{l}\text { SR (with homogeneity) inception } \\
\text { cohort studies; CDR validated in } \\
\text { different populations }\end{array}$ & $\begin{array}{l}\text { SR (with homogeneity) of Level } 1 \\
\text { diagnostic studies; CDR with } 1 \text { b stud- } \\
\text { ies from different clinical centers }\end{array}$ & $\begin{array}{l}\text { SR (with homogeneity) of } \\
\text { prospective cohort studies }\end{array}$ \\
\hline $1 b$ & $\begin{array}{l}\text { Individual RCT (with narrow } \\
\text { Confidence Interval) }\end{array}$ & $\begin{array}{l}\text { Individual inception cohort study } \\
\text { with }>80 \% \text { follow-up; CDR vali- } \\
\text { dated in a single population }\end{array}$ & $\begin{array}{l}\text { Validating cohort study with good } \\
\text { reference standards; or CDR tested } \\
\text { within one clinical centre }\end{array}$ & $\begin{array}{l}\text { Prospective cohort study } \\
\text { with good follow-up }\end{array}$ \\
\hline $2 a$ & $\begin{array}{l}\text { SR (with homogeneity) of } \\
\text { cohort studies }\end{array}$ & $\begin{array}{l}\text { SR (with homogeneity) of either } \\
\text { retrospective cohort studies or } \\
\text { untreated control groups in RCTs }\end{array}$ & $\begin{array}{l}\text { SR (with homogeneity) } \\
\text { of Level > } 2 \text { diagnostic studies }\end{array}$ & $\begin{array}{l}\text { SR (with homogeneity) of } \\
\text { Level } 2 \mathrm{~b} \text { and better studies }\end{array}$ \\
\hline $2 b$ & $\begin{array}{l}\text { Individual cohort study } \\
\text { (including low quality RCT; e. g., } \\
<80 \% \text { follow-up) }\end{array}$ & $\begin{array}{l}\text { Retrospective cohort study or } \\
\text { follow-up of untreated control } \\
\text { patients in an RCT; Derivation of } \\
\text { CDR or validated on split sample } \\
\text { only }\end{array}$ & $\begin{array}{l}\text { Exploratory cohort study with good } \\
\text { reference standards; CDR after } \\
\text { derivation, or validated only on } \\
\text { split-sample or databases }\end{array}$ & $\begin{array}{l}\text { Retrospective cohort study, } \\
\text { or poor follow-up }\end{array}$ \\
\hline $2 c$ & $\begin{array}{l}\text { "Outcomes" Research; } \\
\text { Ecological studies }\end{array}$ & “Outcomes” Research & & Ecological studies \\
\hline $3 a$ & $\begin{array}{l}\text { SR (with homogeneity) of } \\
\text { case-control studies }\end{array}$ & & $\begin{array}{l}\text { SR (with homogeneity) of } 3 \mathrm{~b} \text { and } \\
\text { better studies }\end{array}$ & $\begin{array}{l}\text { SR (with homogeneity) of 3b } \\
\text { and better studies }\end{array}$ \\
\hline $3 b$ & Individual Case-Control Study & & $\begin{array}{l}\text { Non-consecutive study; or without } \\
\text { consistently applied reference } \\
\text { standards }\end{array}$ & $\begin{array}{l}\text { Non-consecutive cohort study; } \\
\text { or very limited population }\end{array}$ \\
\hline 4 & $\begin{array}{l}\text { Case-series (and poor quality } \\
\text { cohort and case-control studies) }\end{array}$ & $\begin{array}{l}\text { Case-series (and poor quality } \\
\text { prognostic cohort studies) }\end{array}$ & $\begin{array}{l}\text { Case-control study, poor or non-in- } \\
\text { dependent reference standard }\end{array}$ & $\begin{array}{l}\text { Case-series or superseded } \\
\text { reference standards }\end{array}$ \\
\hline 5 & $\begin{array}{l}\text { Expert opinion without explicit } \\
\text { critical appraisal, or based on } \\
\text { physiology, bench research or } \\
\text { "first principles" }\end{array}$ & $\begin{array}{l}\text { Expert opinion without explicit } \\
\text { critical appraisal, or based on } \\
\text { physiology, bench research or } \\
\text { "first principles" }\end{array}$ & $\begin{array}{l}\text { Expert opinion without explicit criti- } \\
\text { cal appraisal, or based on physiology, } \\
\text { bench research or "first principles" }\end{array}$ & $\begin{array}{l}\text { Expert opinion without } \\
\text { explicit critical appraisal, or } \\
\text { based on physiology, bench } \\
\text { research or "first principles" }\end{array}$ \\
\hline
\end{tabular}

\subsection{Systematische Literaturrecherchen}

\subsubsection{Suche und Auswahl der Evidenz}

Zu Insgesamt 22 Fragestellungen wurden im Rahmen der Aktualisierung 2017 - 2018 systematische Literaturrecherchen durchgeführt. Berücksichtigt wurden dabei Publikationen seit 2013. Die Suchen wurden in der Medline-Datenbank über die PubMedSuchoberfläche sowie in der Cochrane Library zwischen dem 24.07.2017 und dem 04.08.2017 durchgeführt. Weiterführende Details zu den Recherchen (Suchstrategien, Ein- und Ausschlussgründe, Trefferzahlen, Berücksichtigung von Vorschlägen der Experten) können dem Methodenreport der UserGroup entnommen werden (verfügbar unter https://www.leitlinienprogrammonkologie.de/leitlinien/oesophaguskarzinom/).

\subsubsection{Bewertung und Extraktion der Evidenz}

Die Literaturbewertung wurde - wie schon in der Version 1 - nach der Evidenzklassifizierung des Oxford Centre for Evidence-based Medicine 2009 (siehe $>$ Tab. 1) für Interventions- und diagnostische Studien durchgeführt. Alle eingeschlossenen Studien wurden darüber hinaus in Evidenztabellen extrahiert. Die methodische Qualität der Literaturstelle wurde mit Hilfe von Checklisten überprüft und die gefundenen Mängel wurden im „Notes“ Bereich der Evidenztabellen festgehalten. Als Checklisten wurden die Critical appraisal tools des Oxford Centre for Evidence-based Medicine ${ }^{1}$ bzw. die Newcastle-Ottawa Scale ${ }^{2}$ für nicht-randomisierte Studien (Cohort and Case-control) herangezogen. Studien mit methodischen Schwächen und/ oder bedeutsamer Heterogenität wurden mit einem „-“ nach dem Evidenzgrad markiert. Eine entsprechende Begründung findet sich in der Evidenztabelle im Feld „Notes“. Studien mit einer großen Anzahl gravierender methodischer Mängel konnten unter Angabe einer detaillierten Begründung als Evidenzgrundlage ausgeschlossen werden.

Die Evidenztabellen inklusive der Studienbewertungen können dem Methodenreport der UserGroup entnommen werden (verfügbar unter https://www.leitlinienprogramm-onkologie.de/ leitlinien/oesophaguskarzinom/)
1 CEBM (Centre for Evidence-Based Medicine) Critical Appraisal tools (2017). http://www.cebm.net/critical-appraisal/ (abgerufen am 18.10.2017).

2 Newcastle-Ottawa Scale (NOS) for assessing the quality of nonrandomized studies in meta-analyses (2014) http://www.ohri.ca/programs/ clinical_epidemiology/oxford.asp (abgerufen am 18.10.2017). 


\subsection{Formulierung der Empfehlungen und formale Konsensusfindung}

\subsubsection{Schema der Empfehlungsgraduierung}

Die Methodik des Leitlinienprogramms Onkologie sieht eine Vergabe von Empfehlungsgraden durch die Leitlinienautoren im Rahmen eines formalen Konsensusverfahrens vor. Im Rahmen der Aktualisierung 2017 - 2018 erfolgte nach Diskussion von Änderungsvorschlägen in den Arbeitsgruppen und innerhalb der Steuergruppe eine Online-Abstimmung über das Leitlinienentwicklungsportal der UserGroup (siehe auch Kapitel 5.3.3).

In der Leitlinie werden zu allen evidenzbasierten Statements und Empfehlungen das Evidenzlevel der zugrunde liegenden Studien sowie bei Empfehlungen zusätzlich die Stärke der Empfehlung (Empfehlungsgrad) ausgewiesen. Hinsichtlich der Stärke der Empfehlung werden in dieser Leitlinie drei Empfehlungsgrade unterschieden, die sich auch in der Formulierung der Empfehlungen jeweils widerspiegeln (siehe $>$ Tab. 2, 3).

\subsubsection{Festlegung des Empfehlungsgrades}

Grundsätzlich erfolgte eine Anlehnung der evidenzbasierten Empfehlungen hinsichtlich ihres Empfehlungsgrades an die Stärke der verfügbaren Evidenz, d. h. ein hoher Evidenzgrad (z. B. Metaanalysen/systematische Übersichten von RCTs oder mehrere methodisch hochwertige RCTs), d. h. eine hohe Sicherheit bzgl. der Ergebnisse soll in der Regel auch zu einer starken Empfehlung (Empfehlungsgrad A, „soll“) führen.

Zusätzlich werden weitere Kriterien bei der Wahl des Empfehlungsgrades berücksichtigt. Diese folgenden berücksichtigten Kriterien konnten zu einem Abweichen der Empfehlungsstärke nach oben oder unten führen:

1. Konsistenz der Studienergebnisse

Bsp.: Die Effektschätzer der Studienergebnisse gehen in unterschiedliche Richtungen und zeigen keine einheitliche Tendenz.

2. Klinische Relevanz der Endpunkte und Effektstärken

Bsp.: Es liegen zwar Studien mit Ergebnissen in eine Richtung vor, jedoch wird die Bedeutung der gewählten Endpunkte und/ oder Effektstärken als nicht relevant eingeschätzt.

3. Nutzen-Risiko-Verhältnis Bsp.: Dem nachgewiesenen Nutzen einer Intervention steht ein relevanter Schadensaspekt gegenüber, der gegen eine uneingeschränkte Empfehlung spricht.

4. Ethische Verpflichtungen

Bsp.: Downgrading: Aus ethischen Gründen kann eine Intervention mit nachgewiesenem Nutzen nicht uneingeschränkt angeboten werden. Upgrading: Starke Empfehlung auf Basis von z. B. Fall-Kontroll-Studien, da aus ethischen Gründen ein RCT nicht durchführbar ist.

5. Patientenpräferenzen

Bsp.: Eine Intervention mit nachgewiesenem Nutzen wird nicht stark empfohlen, da sie von den Patienten als belastend oder nicht praktikabel abgelehnt wird.

6. Anwendbarkeit, Umsetzbarkeit in der Versorgung Bsp.: Eine Intervention mit nachgewiesenen positiven Effekten kann nicht empfohlen werden, weil sie im regionalen Versor-
Tab. 2 Schema der Empfehlungsgraduierung.

\begin{tabular}{|l|l|l|}
\hline Empfehlungsgrad & Beschreibung & Ausdrucksweise \\
\hline A & Starke Empfehlung & soll/soll nicht \\
\hline B & Empfehlung & sollte/sollte nicht \\
\hline 0 & Empfehlung offen & $\begin{array}{l}\text { kann/kann verzichtet } \\
\text { werden }\end{array}$ \\
\hline
\end{tabular}

- Tab. 3 Festlegungen hinsichtlich der Konsensstärke.

\begin{tabular}{|l|l|}
\hline Konsensstärke & Prozentuale Zustimmung \\
\hline Starker Konsens & $>95 \%$ der Stimmberechtigten \\
\hline Konsens & $>75-95 \%$ der Stimmberechtigten \\
\hline $\begin{array}{l}\text { Mehrheitliche } \\
\text { Zustimmung }\end{array}$ & $>50-75 \%$ der Stimmberechtigten \\
\hline Dissens & $<50 \%$ der Stimmberechtigten \\
\hline
\end{tabular}

gungssystem aus strukturellen Gründen nicht angeboten werden kann.

\subsubsection{Formale Konsensusverfahren und Konsensuskonferenz}

Nach Abschluss der Evidenzrecherchen wurden die Ergebnisse durch die Steuergruppe primär gesichtet und potentielle Themen für Änderungen oder Ergänzungen identifiziert. In Absprache mit den Arbeitsgruppen wurden anschließend konkrete Änderungsvorschläge formuliert. Im Rahmen der Steuergruppe wurden die Änderungsvorschläge zunächst diskutiert und überarbeitet und anschließend im Rahmen der gesamten Leitliniengruppe formal abgestimmt. Die Abstimmung erfolgte hierbei durch eine elektronische Delphi-Abstimmung über das „UserGroup-Leitlinienportal“ (www.leitlinienentwicklung.de). Empfehlungen mit einer Zustimmung $>75 \%$ und ohne inhaltliche Anmerkungen galten als angenommen.

Da die Steuergruppe keinen Konsens erzielen konnte, wie die Empfehlung zur Dysplasiebeurteilung geändert werden soll, wurden zunächst die beiden Vorschläge der Steuergruppe abgestimmt und anschließend der Vorschlag mit der größten Zustimmung (73,8\%) zusammen mit den restlichen Empfehlungsvorschlägen. Da alle Empfehlungsvorschläge im Rahmen der Online-Abstimmung konsentiert werden konnten, war keine weitere Abstimmungsrunde notwendig. Die Ergebnisse der Online- Abstimmungen können beim OL-Office angefordert werden ( $\vee$ Tab. 4$)$.

\section{Ableitung der Qualitätsindikatoren}

Eine Überarbeitung der Qualitätsindikatoren im Rahmen der Aktualisierung 2017 - 2018 erfolgte nicht. Ausschlaggebend hierfür waren die folgenden Punkte: 
- Tab.4 Zeitlicher Ablauf der Aktualisierung 2017 - 2018.

\begin{tabular}{|c|c|c|}
\hline Prozess & Datum & Agenda/Themen \\
\hline Treffen der Steuergruppe & 05.07.2017 & $\begin{array}{l}\text { Abstimmung der Themenkomplexe und Fragen, Definition von Schlüsselfragen. } \\
\text { Diskussion des organisatorischen Ablaufs inklusive systematischer Literaturrecher- } \\
\text { che und Evidenzbeurteilung, Besprechung der Schlüsselfragen. } \\
\text { Konstituierung der Arbeitsgruppen mit AG-Leitern, Konstituierung der Steuergruppe. }\end{array}$ \\
\hline Literaturrecherche Evidenzbeurteilung & $07 / 2017-12 / 2017$ & $\begin{array}{l}\text { Information aller Fachexperten } \\
\text { Definition des Zeitraums, für den Literatur gesucht werden soll. Installation des } \\
\text { Leitlinienportals. Anlage einer Referenzdatenbank und einer pdf-Bibliothek für } \\
\text { Literatur (Volltexte). Vervollständigung der Suche nach existierenden Leitlinien. } \\
\text { Bewertung der bisherigen Leitlinien nach DELBI, auch hinsichtlich der klinischen } \\
\text { Verwendbarkeit/Aktualität/Übertragbarkeit auf Deutschland. } \\
\text { Erstellung von Evidenztabellen }\end{array}$ \\
\hline Bearbeitung in der Steuergruppe & $01 / 2018-04 / 2018$ & $\begin{array}{l}\text { Vorstellen der Rechercheergebnisse und Leitlinienbewertung Sichtung der } \\
\text { Evidenztabellen Verfeinerung der Literaturrecherche Diskussion der Ergebnisse in } \\
\text { den Arbeitsgruppen Erstellung von Textentwürfen für Hintergrundtexte und } \\
\text { Empfehlungen Ggf. zusätzliche Telefonkonferenzen }\end{array}$ \\
\hline Treffen der Steuergruppe & 30.05 .2018 & $\begin{array}{l}\text { Diskussion der Textentwürfe und Konsentierung der Änderungen } \\
\text { Diskussion des weiteren organisatorischen Ablaufs. }\end{array}$ \\
\hline Redaktion und Konsentierung & 05. -09.2018 & $\begin{array}{l}\text { Erstellung der überarbeiteten Langversion, Konsentierung der Empfehlungen, } \\
\text { Erstellung der Konsultationsfassung von Langversion und Leitlinienreport. }\end{array}$ \\
\hline
\end{tabular}

1. Im Rahmen der Aktualisierung wurde eine modifizierte starke Empfehlung (6.18) erarbeitet, die wenig Erfolg hat, eine Qualitätsindikator zu werden.

2. Die Empfehlungen, die die Grundlage für die aktuellen Q। bilden, sind unverändert geblieben.

3. Bisher liegen keine Daten zu den bisherigen QI vor. Entsprechende Daten aus den DKG-zertifizierten Zentren werden für 2019 erwartet.

Auf der Grundlage der ersten Daten aus den zertifizierten Zentren und einer aktualisierten internationalen Recherche nach Qualitätsindikatoren wird voraussichtlich 2019 eine Aktualisierung der Qualitätsindikatoren erfolgen.

\section{Reviewverfahren und Verabschiedung}

Während der Aktualisierungsprozesses erfolgten methodische Reviews durch das OL-Office und nach Abschluss der Konsentierung durch das AWMF-IMWi. Im Anschluss werden ein öffentliches Konsultationsverfahren und die formale Zustimmung der beteiligten Fachgesellschaften/Organisationen durchgeführt.

Die Konsultationsfassung der Leitlinie konnte vom 11.10. bis zum 21.11. kommentiert werden. Im Rahmen der Konsultation gingen lediglich zwei Kommentare ein:

1. Redaktioneller Hinweis zur korrekten Verwendung von Fachbegriffen (Tumoren statt Tumore; Veneninvasion statt Blutgefäßinfiltration)

2. Hinweis, dass ein Kapitel zur Nachsorge ergänzt werden sollte.

Nach Abschluss der Konsultation ging ein Hinweis zu einer fehlerhaften Zitation im Kapitel 4.1. Rauchen ein: Es wurden Effektmaße für Alkoholkonsum aus der Studie von Lee et al. 2007 [1] genannt.
Die redaktionellen Hinweise wurden in der Version 2.0 umgesetzt. Der Vorschlag, ein Kapitel zur Nachsorge zu ergänzen, wird im Rahmen der 2019 geplanten Bedarfsanalyse in der Leitliniengruppe diskutiert werden.

\section{Unabhängigkeit und Umgang mit Interessenkonflikten}

Die Deutsche Krebshilfe stellte über das Leitlinienprogramm Onkologie (OL) finanzielle Mittel für insgesamt drei Jahre zur Verfügung (2017/18 - 2020). Für die Aktualisierung 2018 wurden Mittel für Büromaterial, Literaturbeschaffung/Evidenzbeurteilungen und die Sitzungen der Steuergruppe (Raummieten, Technik, Verpflegung, Reisekosten der Teilnehmer) verwendet.

Die Erarbeitung der Leitlinie erfolgte in redaktioneller Unabhängigkeit von der finanzierenden Organisation. Die Leitliniengruppenteilnehmer arbeiteten ehrenamtlich.

Die Leitliniengruppenmitglieder legten während des Leitlinienprozesses vor der formalen Abstimmung der Empfehlungen eine schriftliche Erklärung zu Interessen anhand des AWMF Formblattes Stand 17.01.2018 (siehe Kapitel 11.1) vor. Die offengelegten Interessen sind in $>$ Tab. 5 aufgeführt.

Für die Organisation der Aktualisierung war zusammen mit dem Koordinator eine interdisziplinär zusammengesetzte Steuergruppe aus Klinikern und Methodikern verantwortlich. In dieser wurden alle Schritte der Aktualisierung besprochen. Bei einem Treffen der Steuergruppe am 05.07.2017 wurde nach interner Diskussion folgendes Procedere zur Bewertung von und zum Umgang mit den offengelegten Interessen festgelegt: 
1. Bei positiven Angaben in den Rubriken: Berater- bzw. Gutachtertätigkeit oder bezahlte Mitarbeit in einem wissenschaftlichen Beirat eines Unternehmens der Gesundheitswirtschaft (z. B. Arzneimittelindustrie, Medizinproduktindustrie), eines kommerziell orientierten Auftragsinstituts oder einer Versicherung oder Besitz von Geschäftsanteilen, Aktien, Fonds mit Beteiligung von Unternehmen der Gesundheitswirtschaft oder Persönliche Beziehungen zu einem Vertretungsberechtigten eines Unternehmens erfolgte eine Bewertung in gering, moderat und hoch.

2. Angaben der Rubriken Vortrags- und Schulungstätigkeit bzw. Wissenschaftlicher Schwerpunkt/Studien wurden in dieser Gruppe grundsätzlich als gering bewertet.

3. Bei Vorliegen eines geringen Interessenkonflikts wurden aufgrund der vorhandenen protektiven Faktoren (unabhängige Evidenzsuche und -bewertung, formales Konsensverfahren, Einbeziehen aller relevanten Stakeholder) keine weiteren Maßnahmen als erforderlich erachtet.

4. Bei Vorliegen eines moderaten Interessenkonflikts erfolgte eine Abstimmungsenthaltung und Doppelabstimmung, um festzustellen, ob die Enthaltung zu einer geänderten Zustimmungsquote führen würde.

5. Wenn aufgrund der offengelegten Sachverhalte in diesen Kategorien ein hohes Risiko für einen Interessenkonflikt angenommen werden musste, sollten diese Personen nicht an der Abstimmung und der Diskussion der entsprechenden Empfehlungen teilnehmen.

Die Beurteilung wurde durch eine Kommission der Leitliniengruppe aus drei Klinikern (Hölscher (DGCH), Hollerbach (DGVS), Vanhoefer (DGHO)) und zwei Methodikern (Langer (OL-Office), Nothacker (AWMF)) vorgenommen. Die Beurteilung 2018 erfolgte hin Hinblick auf die 2018 in der Leitliniengruppe diskutierten und abgestimmten Themen (siehe Seite 2 der Langversion 2.0).

Nach Sichtung der eingegangenen Rückmeldungen der Fachexperten - und wenn erforderlich persönlicher Rücksprache wurde für die Aspekte „computergestützte digitale Filter“ für die Chromoendoskopie bei Autoren aufgrund von Tätigkeit in Advisory Boards von Endoskopiefirmen für dieses Thema ein moderater Interessenkonflikt gesehen und eine Doppelabstimmung avisiert, eine solche Tätigkeit lag jedoch nicht vor. Weiterhin wurde zusätzlich zu den oben genannten Konstellationen für die Empfehlung zur Referenzpathologie ein moderater Interessenkonflikt bei den Vertretern des Berufsverbandes der Pathologen gesehen, hier erfolgte eine Kontrolle des Abstimmungsergebnisses bei Enthaltung der Person. Es ergab sich keine Änderung des Konsenses.

An dieser Stelle möchten wir allen Experten und Mitarbeitern für ihre ausschließlich ehrenamtliche Mitarbeit an dem Projekt danken.

\section{Verbreitung und Implementierung}

Folgende Aktivitäten dienen zur Verbreitung und Implementierung der Leitlinie:

a) Verfügbarmachung einer Patientenleitlinie

b) Erstellung der Qualitätsindikatoren c) Publikation der Leitliniendokumente auf den Internetseiten von AWMF, Leitlinienprogramm-Onkologie und DGVS

d) Publikation der aktualisierten Leitlinieninhalte in Fachzeitschriften

e) Bundesweite Fortbildungsveranstaltungen

f) Vorstellung auf Fachkongressen

\section{Tabellenverzeichnis}

- Tab. 1: Schema der Evidenzgraduierung nach Oxford (Version März 2009)

- Tab.2: Schema der Empfehlungsgraduierung

Tab. 3: Festlegungen hinsichtich der Konsensstark

- Tab. 3: Festlegungen hinsichtlich der Konsensstärke

- Tab. 4: Zeitlicher Ablauf der Aktualisierung 2017 - 2018

- Tab. 5: Ergebnisse der Interessenkonflikterklärungen e107 e107 e108 e112

\section{Anlagen}

\subsection{Formblatt der AWMF zur Erklärung von Interessenkonflikten (Stand: 17.01.2018)}

\section{Erklärung von Interessen}

(Titel, AWMF-Registernummer)

zu Händen

(Leitlinienkoordinator)

\section{Vorbemerkung}

Alle Mitglieder der Leitliniengruppe sind gehalten, die nachstehende Erklärung von Interessen auszufüllen. Die Erklärung wird gegenüber dem Leitlinienkoordinator abgegeben. Dies soll bereits zu Beginn des Leitlinienprojekts erfolgen bzw. zu dem Zeitpunkt, an dem die Mitglieder ihre Teilnahme am Leitlinienprojekt gegenüber dem Koordinator bestätigen. Bei länger andauernden Projekten ist eine Erneuerung der Erklärung einmal pro Jahr bis zum Abschluss der Leitlinienentwicklung, zumindest aber vor der Konsensfindung, erforderlich.

In der Erklärung sind alle Interessen aufzuführen, unabhängig davon, ob der/die Erklärende selbst darin einen thematischen Bezug zur Leitlinie oder einen Interessenkonflikt sieht oder nicht. Ob Interessenkonflikte bestehen und ob dadurch die erforderliche Neutralität für die Mitarbeit bei der Leitlinienentwicklung in Frage gestellt ist oder in welchen speziellen Bereichen/Fragestellungen der Leitlinie das professionelle Urteilsvermögen eines Experten durch sekundäre Interessen beeinflusst sein könnte, ist durch einen Dritten zu bewerten und in der Leitliniengruppe zu diskutieren. Die Erklärung betrifft Interessen innerhalb des laufenden Jahres sowie der zurückliegenden 3 Jahre.

Die Originale der Erklärungen verbleiben vertraulich beim Leitlinienkoordinator. Die Inhalte der Erklärungen sind in der Langfassung der Leitlinie bzw. im Leitlinienreport in standardisierter Zusammenfassung offen darzulegen. Ergänzend sind das Verfahren der Sammlung und Bewertung der Erklärungen sowie die Ergebnisse der Diskussion zum Umgang mit Interessenkonflikten darzulegen. 


\section{Erklärung}

\section{Allgemeine Angaben}

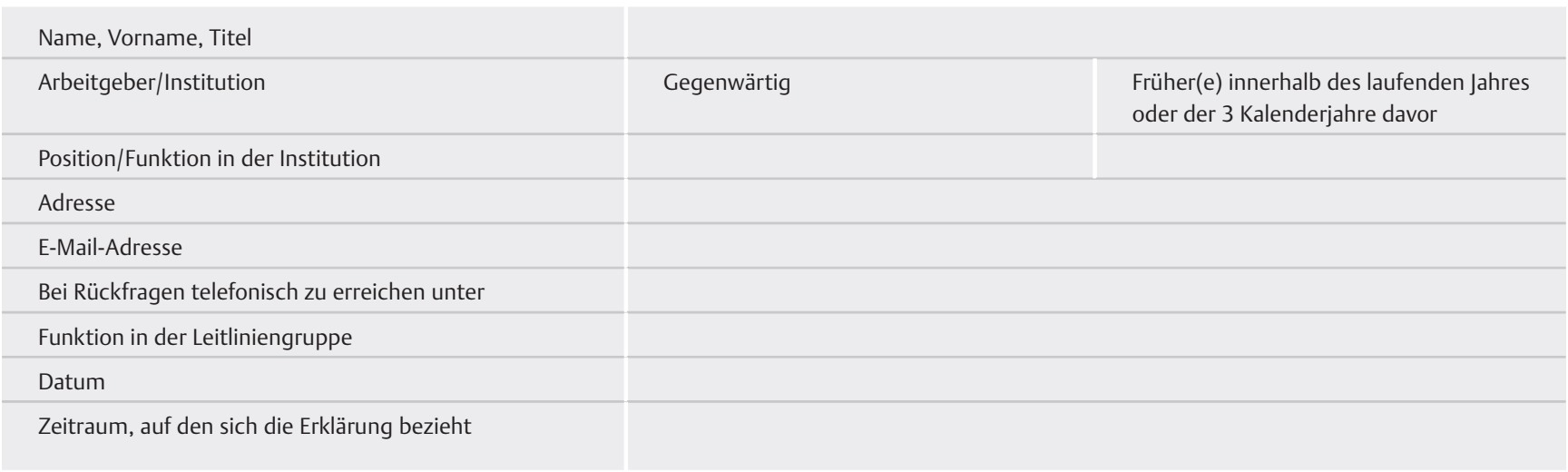

\section{Direkte, finanzielle Interessen}

Hier werden finanzielle Beziehungen zu Unternehmen, Institutionen oder Interessenverbänden im Gesundheitswesen erfasst. Haben Sie oder die Einrichtung, für die Sie tätig sind, innerhalb des laufenden Jahres oder der 3 Kalenderjahre davor Zuwendungen erhalten von Unternehmen der Gesundheitswirtschaft (z. B. Arzneimittelindustrie, Medizinproduktindustrie), industriellen

Interessenverbänden, kommerziell orientierten Auftragsinstituten, Versicherungen/Versicherungsträgern oder von öffentlichen Geldgebern (z. B. Ministerien), Körperschaften/Einrichtungen der Selbstverwaltung, Stiftungen oder anderen Geldgebern? Machen Sie bitte in folgender Tabelle zu allen zutreffenden Aspekten konkrete Angaben.

\begin{tabular}{|c|c|c|c|c|c|c|}
\hline Art der Beziehung/Tätigkeit & $\begin{array}{l}\text { Name des/der } \\
\text { Kooperations- } \\
\text { partner/s }\end{array}$ & $\begin{array}{l}\text { Zeitraum der } \\
\text { Beziehung/ } \\
\text { Tätigkeit }^{1}\end{array}$ & $\begin{array}{l}\text { Thema, Bezug } \\
\text { zur Leitlinie }^{2}\end{array}$ & $\begin{array}{l}\text { Art der } \\
\text { Zuwendung }\end{array}$ & $\begin{array}{l}\text { Höhe der } \\
\text { Zuwendung }\end{array}$ & Empfänger $^{5}$ \\
\hline \multicolumn{7}{|l|}{ Berater-/Gutachtertätigkeit } \\
\hline \multicolumn{7}{|l|}{$\begin{array}{l}\text { Mitarbeit in einem Wissenschaft- } \\
\text { lichen Beirat (advisory board) }\end{array}$} \\
\hline \multicolumn{7}{|l|}{$\begin{array}{l}\text { Vortrags- oder } \\
\text { Schulungstätigkeit }\end{array}$} \\
\hline \multicolumn{7}{|l|}{ Autoren- oder Coautorenschaft } \\
\hline \multicolumn{7}{|l|}{$\begin{array}{l}\text { Forschungsvorhaben/ } \\
\text { Durchführung klinischer } \\
\text { Studien }\end{array}$} \\
\hline \multicolumn{7}{|l|}{$\begin{array}{l}\text { Eigentümerinteressen (Patent, } \\
\text { Urheberrecht, Aktienbesitz }{ }^{6} \text { ) }\end{array}$} \\
\hline \multicolumn{7}{|c|}{$\begin{array}{l}1 \text { Innerhalb des Erfassungszeitraums, d. h.im gegenwärtigen und den zurückliegenden } 3 \text { Jahren, Angabe: von (Monat/Jahr) bis (Monat/Jahr). } \\
2 \text { Angabe des Themas, bei Präparaten/Geräten auch Handelsname bzw. Wirkstoffname (Freitext), zusätzlich Angabe einer Selbsteinschätzung des Bezugs } \\
\text { zur Leitlinie: „Nein“ oder „Ja“. } \\
{ }^{3} \text { Honorar, Drittmittel, geldwerte Vorteile (z. B. Personal- oder Sachmittel; Reisekosten, Teilnahmegebühren, Bewirtung i. R. von Veranstaltungen), Verkaufslizenz. } \\
{ }^{4} \text { Es können gerundete Beträge angeben werden (z. B. bei Beiträgen > } 1000 € \text { jeweils auf die nächste Tausenderstelle): Die Angaben beziehen sich auf die } \\
\text { Gesamtsumme der Zuwendungen für eine angegebene Tätigkeit über den Erfassungszeitraum, Angabe: von (Monat/Jahr) bis (Monat/Jahr). Diese Anga- } \\
\text { ben werden vertraulich behandelt. }\end{array}$} \\
\hline
\end{tabular}




\section{Indirekte Interessen}

Hier werden persönliche Beziehungen zu Interessenverbänden im Gesundheitswesen, „intellektuelle“, akademische und wissenschaftliche Interessen oder Standpunkte sowie Schwerpunkte klinischer Tätigkeiten/Einkommensquellen erfasst (für den Zeitraum des laufenden Jahres oder der 3 Kalenderjahre davor). Hierunter fallen auch solche, die indirekt mit finanziellen persönlichen Interessen verbunden sein können.

- Sind oder waren Sie in Wissenschaftlichen Fachgesellschaften, Berufsverbänden, Institutionen der Selbstverwaltung, Patientenselbsthilfegruppen, Verbrauchervertretungen oder anderen Verbänden aktiv? Wenn ja, in welcher Funktion (z. B. Mandatsträger für diese/andere Leitlinien, Vorstand)?
- Können Sie Schwerpunkte Ihrer wissenschaftlichen und/oder klinischen Tätigkeiten benennen? Fühlen Sie sich bestimmten „Schulen“ zugehörig?

- Waren Sie an der inhaltlichen Gestaltung von Fortbildungen federführend beteiligt?

- Haben Sie persönliche Beziehungen (als Partner oder Verwandter 1. Grades) zu einem Vertretungsberechtigten eines Unternehmens der Gesundheitswirtschaft?

Machen Sie bitte in folgender Tabelle zu allen zutreffenden Aspekten konkrete Angaben.

\begin{tabular}{|c|c|c|c|}
\hline Art der Beziehung/Tätigkeit & $\begin{array}{l}\text { Namen/Schwerpunkte } \\
\text { (bitte konkret benennen) }\end{array}$ & $\begin{array}{l}\text { Zeitraum der Beziehung/ } \\
\text { Tätigkeit' }^{1}\end{array}$ & $\begin{array}{l}\text { Themenbezug zur } \\
\text { Leitlinie }^{2}\end{array}$ \\
\hline \multicolumn{4}{|l|}{ Mitgliedschaft/Funktion in Interessenverbänden } \\
\hline \multicolumn{4}{|l|}{ Schwerpunkte wissenschaftlicher Tätigkeiten, Publikationen } \\
\hline \multicolumn{4}{|l|}{ Schwerpunkte klinischer Tätigkeiten } \\
\hline \multicolumn{4}{|l|}{ Federführende Beteiligung an Fortbildungen/Ausbildungsinstituten } \\
\hline $\begin{array}{l}\text { Persönliche Beziehungen (als Partner oder Verwandter 1. Grades) } \\
\text { zu einem Vertretungsberechtigten eines Unternehmens der } \\
\text { Gesundheitswirtschaft }\end{array}$ & & & \\
\hline
\end{tabular}

\section{Sonstige Interessen}

Sehen Sie andere Aspekte oder Umstände, die von Dritten als einschränkend in Bezug auf Ihre Objektivität oder Unabhängigkeit wahrgenommen werden könnten?

Ich erkläre hiermit nach bestem Wissen und Gewissen, dass ich alle mir derzeit bekannten Umstände aufgeführt habe, die gegebenenfalls zu einem persönlichen Interessenkonflikt bei der themenbezogenen Mitwirkung bei der Erstellung der Leitlinie führen können. Ich erkläre weiterhin, dass ich die Diskussion der Erklärungen anderer Mitglieder in der Leitliniengruppe absolut vertraulich behandeln werde. Ich bin darüber informiert, dass die Angaben in standardisierter Zusammenfassung mit der Leitlinie/in einem begleitenden Leitlinienreport veröffentlicht werden und dass das vorliegende Formular vor der Einsicht unberechtigter Dritter geschützt aufbewahrt wird. Hiermit bin ich einverstanden.

Datum

Unterschrift

\section{Ergänzende Hinweise}

- Bitte füllen Sie das Formular vollständig aus.

- Falls Sie zu bestimmten Fragen keine Angaben machen können oder wollen, begründen Sie dies bitte.

- Bitte speichern Sie das ausgefüllte Formular und senden es an das Leitliniensekretariat: xxx@yyy.zz 


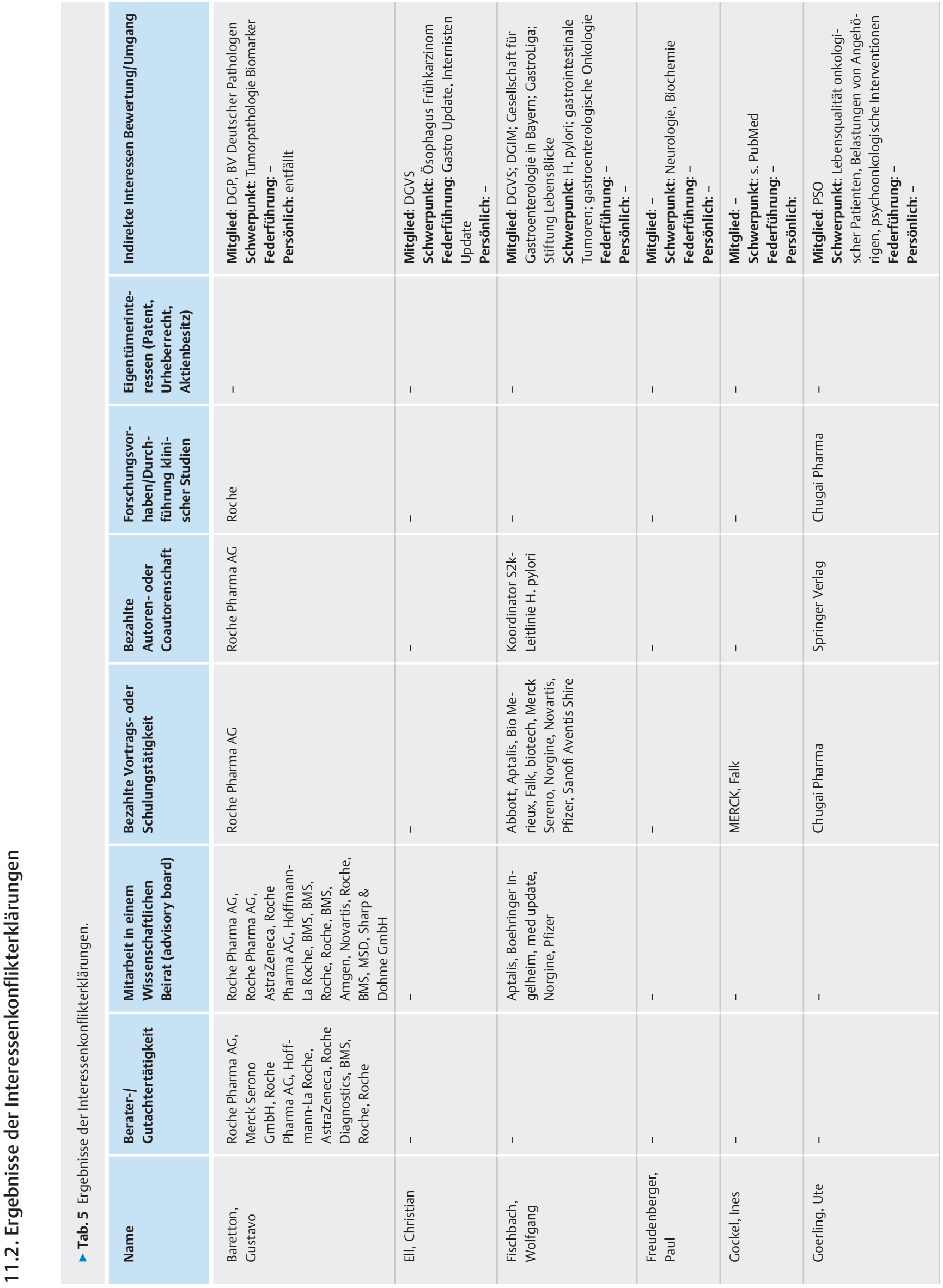




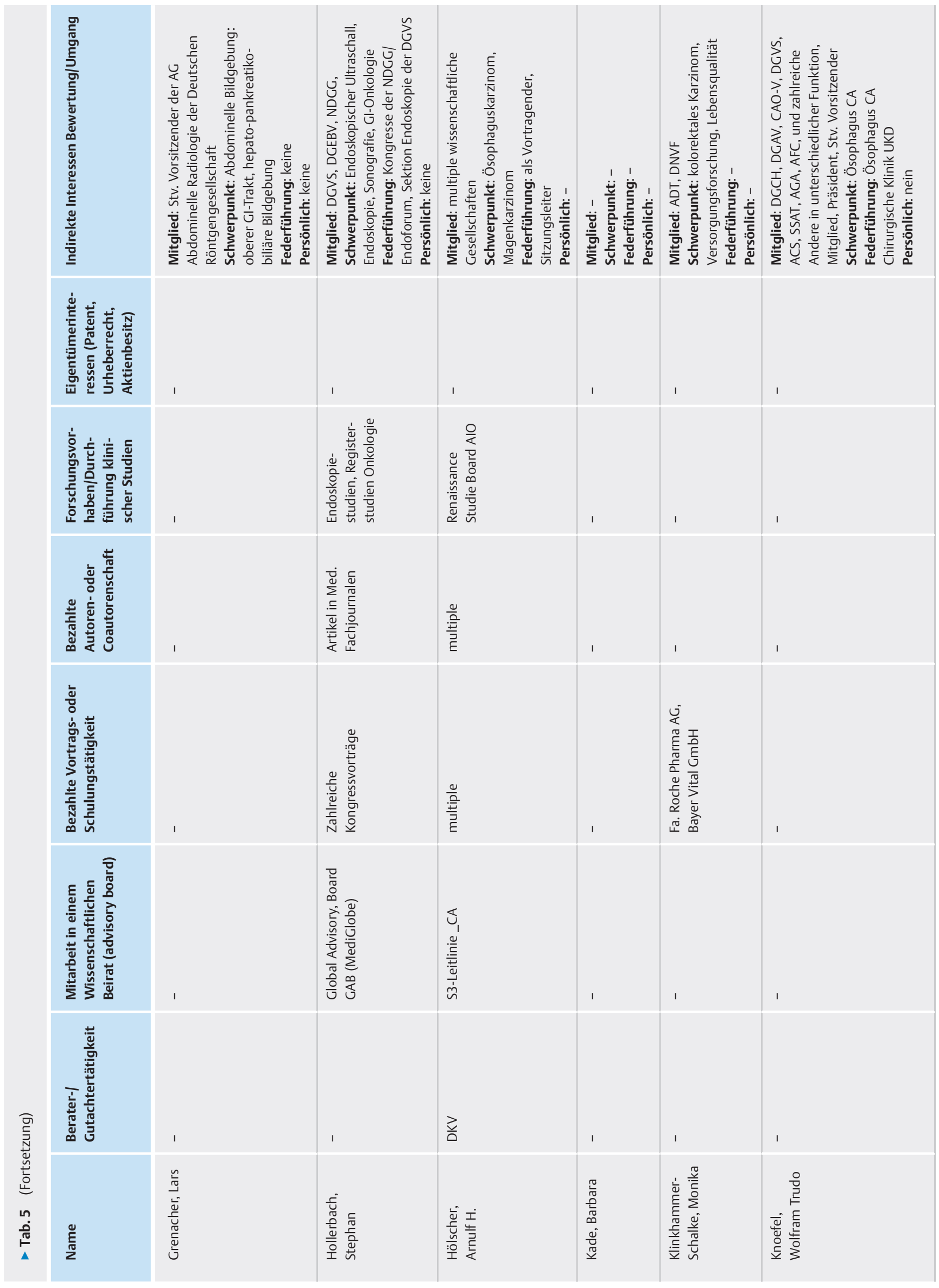




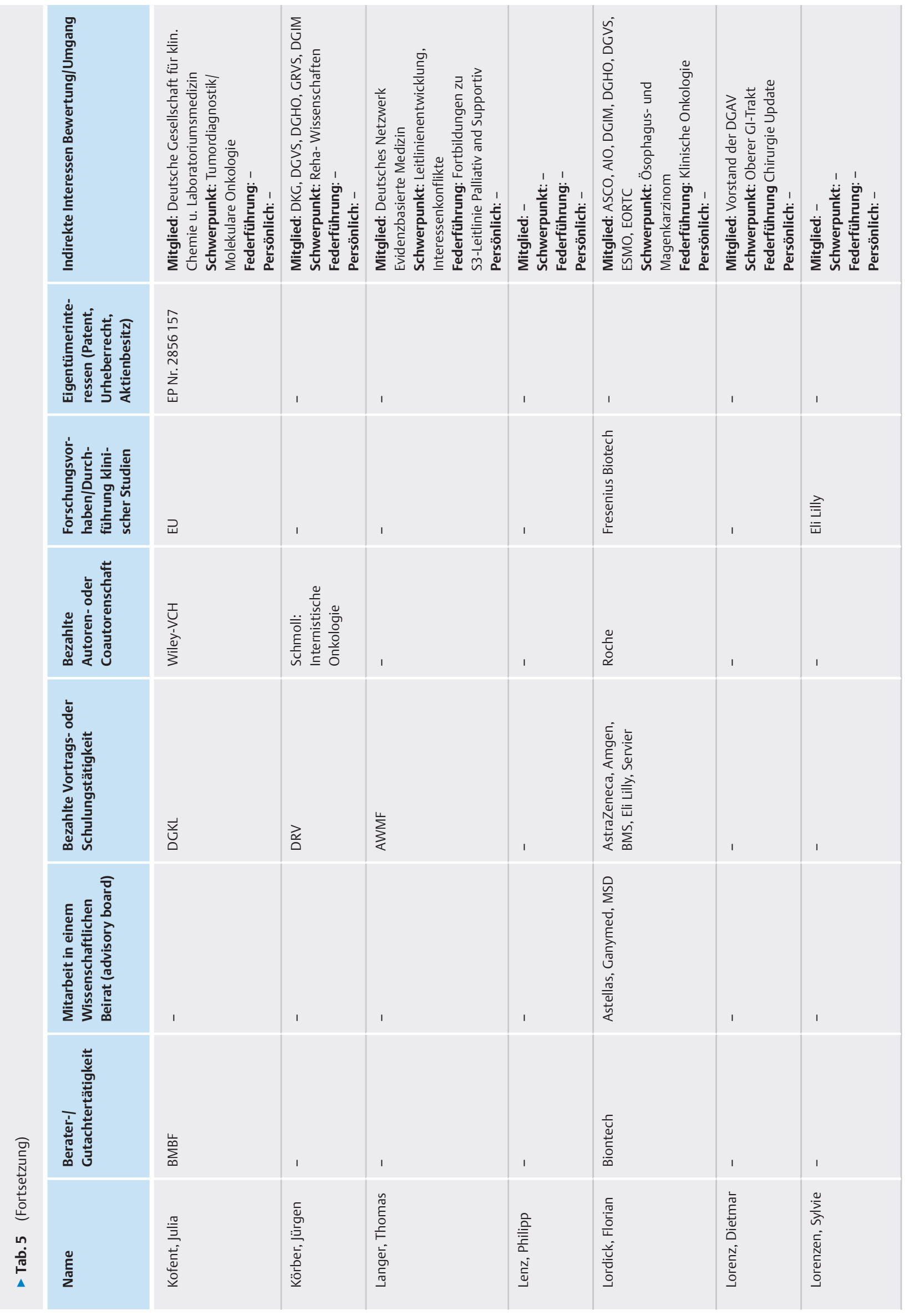




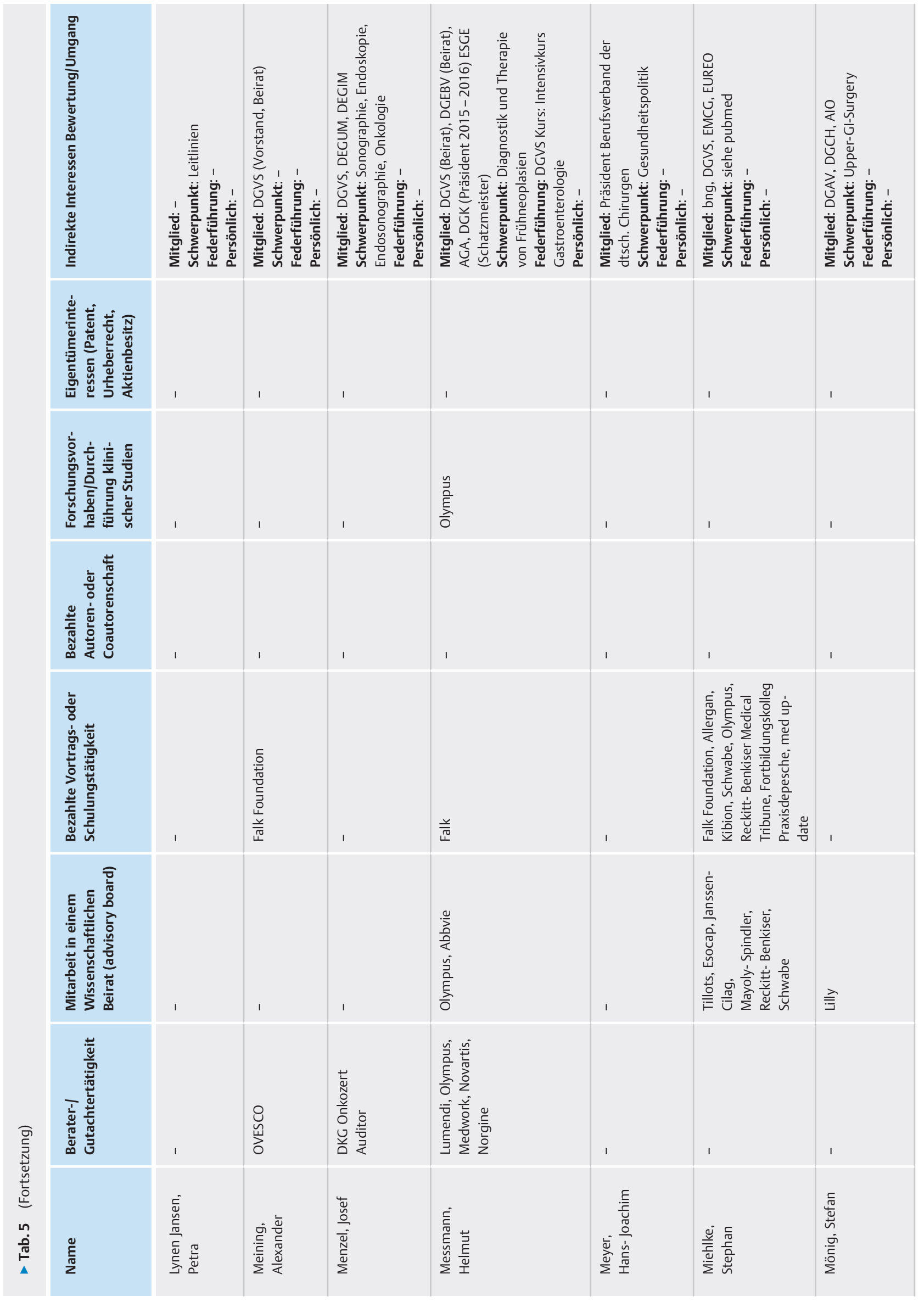




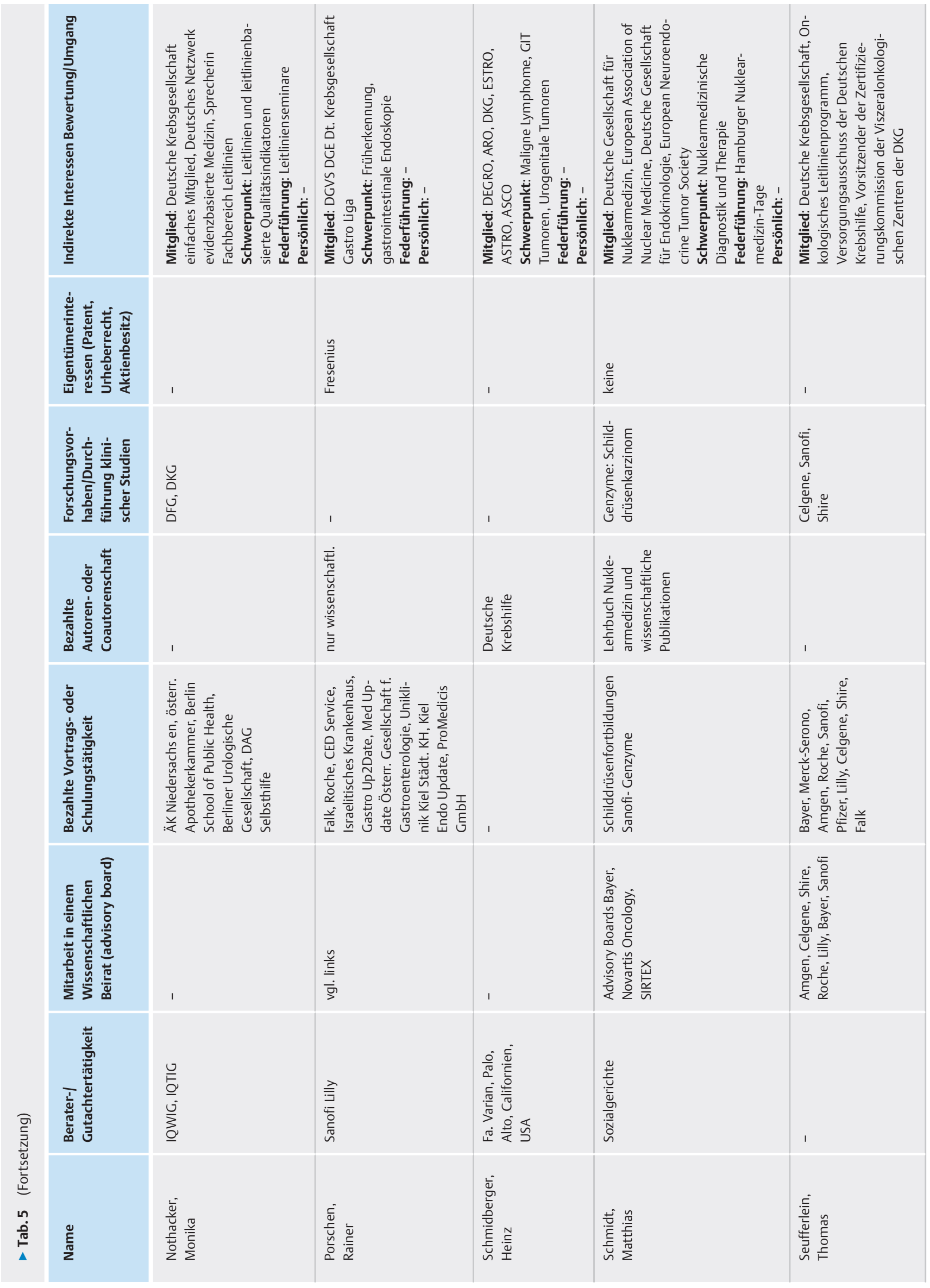




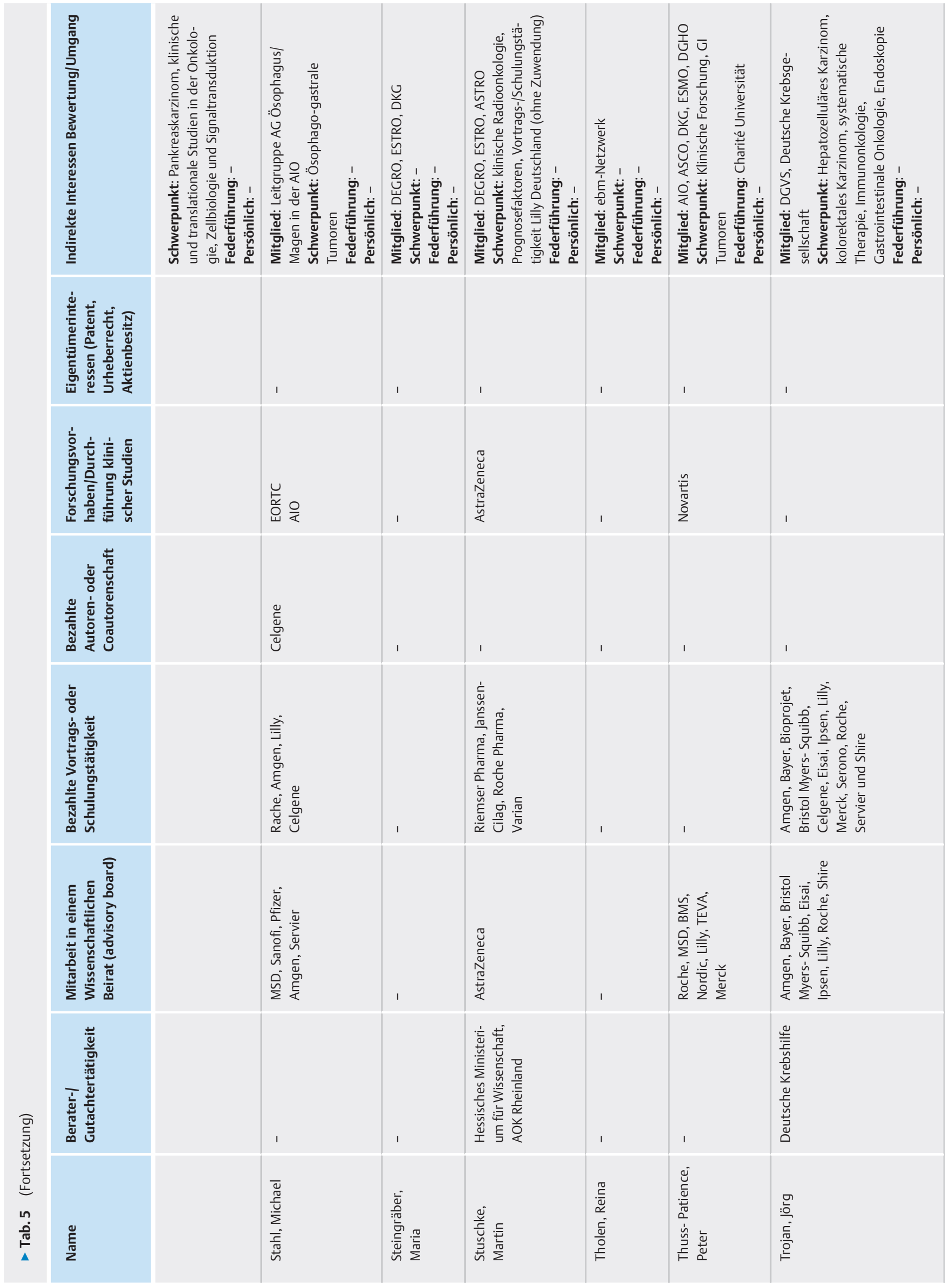




\begin{tabular}{|c|c|c|c|c|c|c|}
\hline 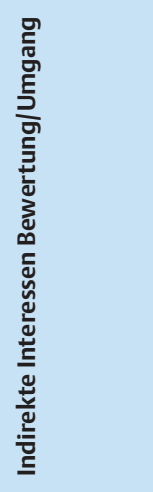 & 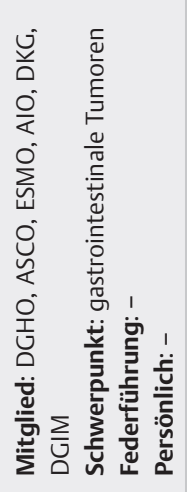 & 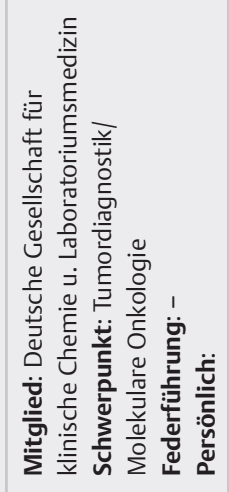 & 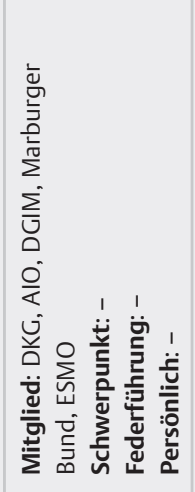 & 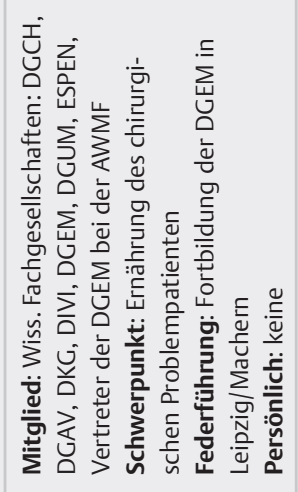 & 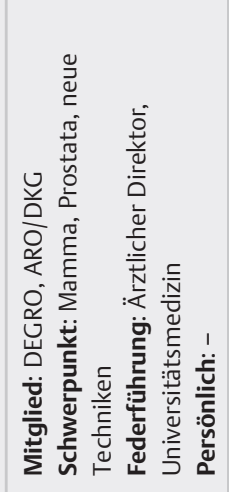 & 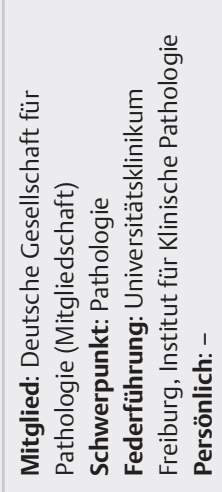 \\
\hline 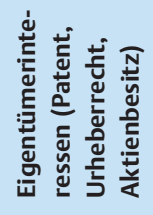 & I & 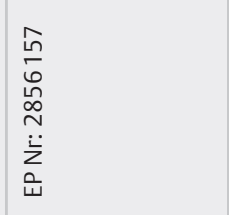 & 1 & I & $\stackrel{\tilde{\tilde{N}}}{N}$ & $\dot{z}$ \\
\hline 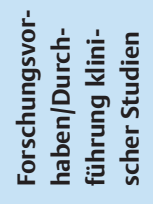 & 1 & 己 & 1 & 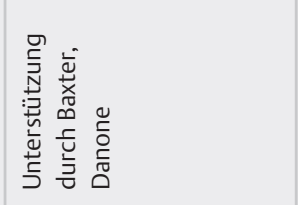 & 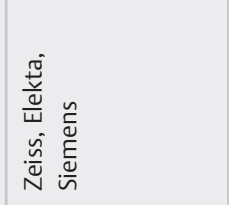 & $\stackrel{ \pm}{\bar{y}}$ \\
\hline 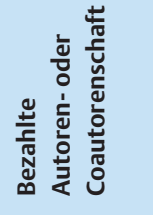 & 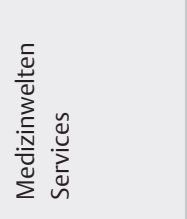 & 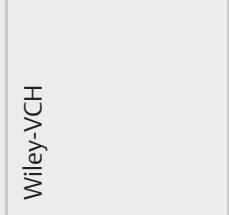 & 1 & 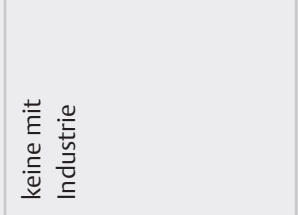 & 1 & $\stackrel{\geq}{ \pm}$ \\
\hline 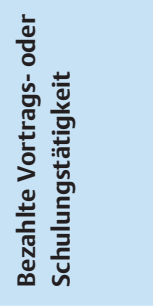 & 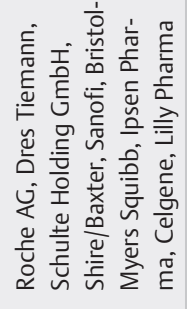 & 咅 & 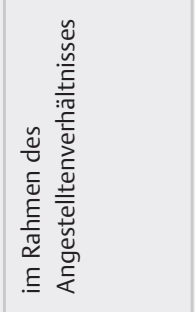 & 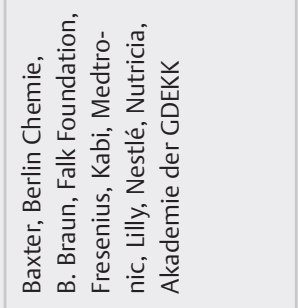 & 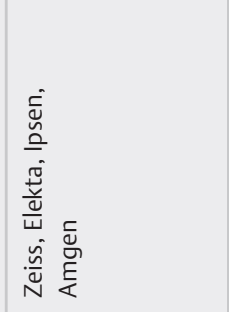 & 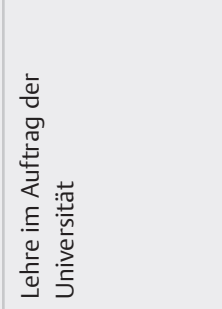 \\
\hline 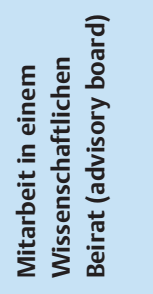 & 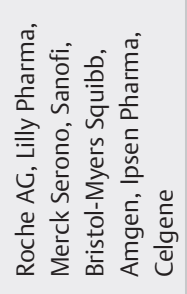 & 1 & 1 & 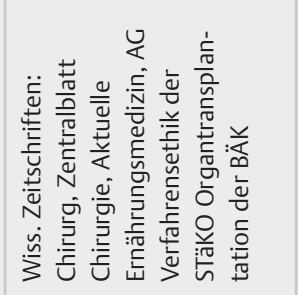 & 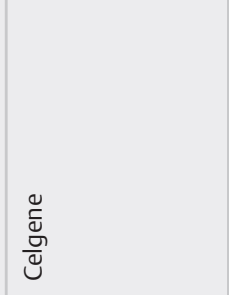 & 1 \\
\hline 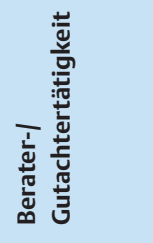 & 1 & 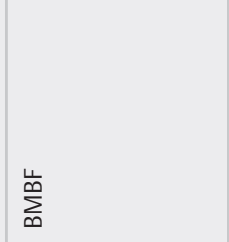 & 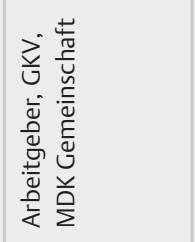 & 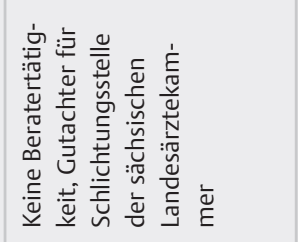 & 1 & 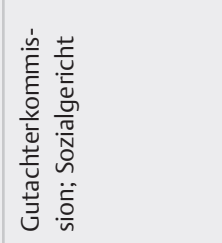 \\
\hline $\begin{array}{l}\text { 足 } \\
\frac{\text { N0 }}{2}\end{array}$ & 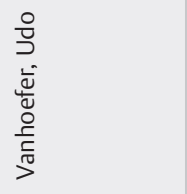 & 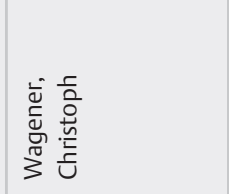 & 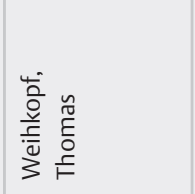 & 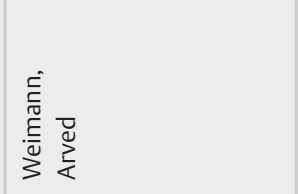 & 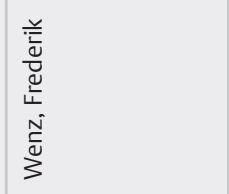 & 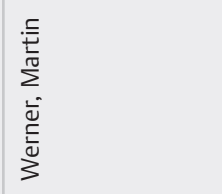 \\
\hline
\end{tabular}




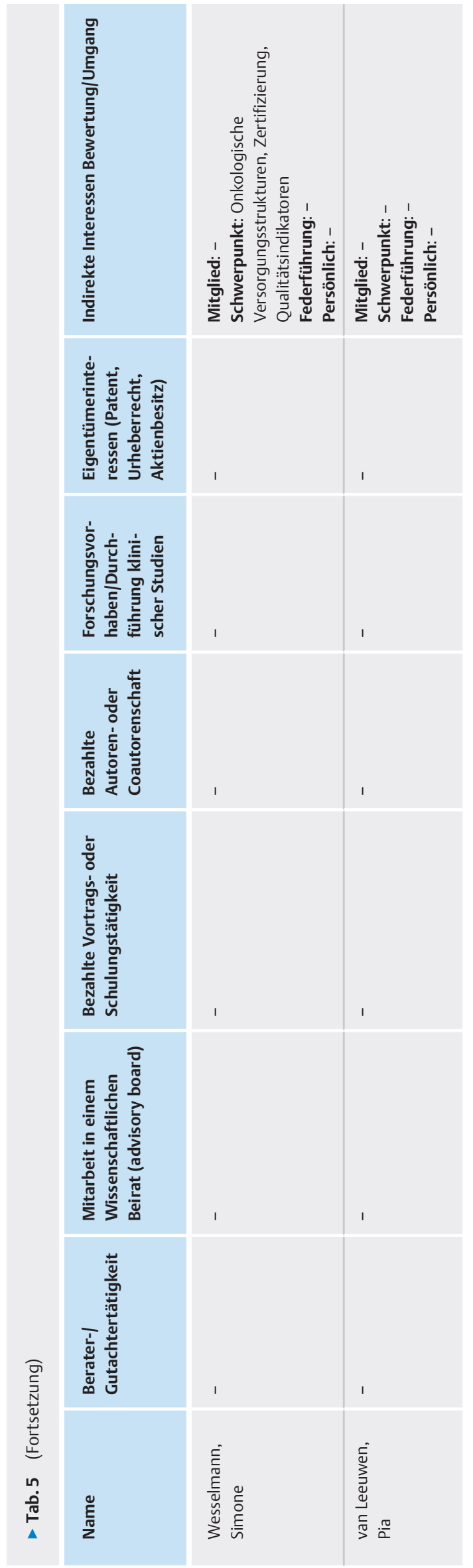

Interessenkonflikt

Die Autoren geben an, dass kein Interessenkonflikt besteht.

Literatur

[1] Lee $\mathrm{CH}$ et al. Anatomical subsite discrepancy in relation to the impact of the consumption of alcohol, tobacco and betel quid on esophageal cancer. Int J Cancer 2007; 120: 1755-1762 